\section{Breslow replies to criticism}

SIR - I have just read the complaint by Menger and Haim (Nature 359, 666$668 ; 1992)$ that they were poorly treated by J. Am. chem. Soc. (JACS) in their attempt to criticize some work we published a few years ago. The situation is not as they represent it. Menger's paper was rejected by $J A C S$ because of referees' reports that it lacked merit; I was not a referee, but the referees saw my comments. Among other flaws, it misrepresented what we had published, then attacked the misrepresentation. It was eventually published in $J$. org. Chem. $(J O C)$, leading a $J A C S$ editor to demand in writing to the $J O C$ editor that the Menger paper be retracted because of its flaws, particularly the misrepresentations.

The main complaint in Menger's paper was that we had used the phrase "negative experimental catalytic rate constant" to describe our observation that one of the reactions slows as more catalyst is added. The phenomenon is not unusual (it is related to the well-known common ion effect in solvolysis) and is completely confirmed by our later work. It is seen because an intermediate partitions along two different pathways; thus speeding up one path with the catalyst slows the rate of formation of the other product. This proves a common intermediate for the two processes. Even though one would certainly refer to the coefficient of a catalyst as an "experimental catalytic rate constant" for any process that speeds up when catalyst is added, some chemists object to calling it an experimental rate constant when it is a negative number. This is a matter of taste, not an error. The original data, and our new work, fully confirm the fact of the rate decrease. Of course we never invoked negative rates, simply a negative catalytic coefficient to describe quantitatively the slowing of the reaction.

The Haim paper is apparently to be published in $J A C S$. It was originally rejected after referees' comments, but eventually accepted. It claims that our original data do not fit very well the expected kinetics for the mechanism we propose, but does not propose an alternative mechanism.

Since that time we have done new work, currently being refereed by JACS. Haim and Menger have both seen this new paper. It completely confirms our previous conclusions, including the negative catalytic effect, and shows that a sophisticated kinetic treatment fully accommodates the previous data as well. It also calls attention to our first published work on this system which had many carefully buffered data points. With this foundation, fewer data were

NATURE · VOL 360 - 5 NOVEMBER 1992 needed for the later work that has been criticized as being incomplete. The new extensive work and the sophisticated kinetic treatment have been presented before experts at meetings such as the reaction mechanism symposium, and have met no objections. The scientific facts fully support our previous conclusions.

Of course science must be selfcorrecting, but criticism should be well founded. There is no excuse for distorting what has been published in order to attack it. The happy ending to this story is that we were stimulated to do our new studies by outrage over the misrepresentations. This new work has added considerably to our understanding while confirming the previous conclusions. The overall result has let us reinterpret the mechanism of an important enzyme, a reinterpretation supported by others, and it has guided us to the design of an improved enzyme mimic.

\section{Ronald Breslow}

Department of Chemistry,

Columbia University,

New York, New York 10027, USA

\section{Coley's toxins}

SIR - Brouckaert et al. ${ }^{1}$, in response to my Commentary ${ }^{2}$, discuss several issues that need to be addressed. First, the optimistic clinical results mentioned by Brouckaert et al., while very impressive and important in their own right, all involve some form of direct, local administration of cytokines within or near the site of the growing tumour. The ability to make such a delivery was a luxury not shared by most of the inoperable softtissue sarcoma patients treated by Coley and his contemporaries, nor would it be shared by those patients with similar disease today. It is also not necessary for the successful treatment of a truly sensitive murine tumour (such as Meth A).

Second, Brouckaert et al. assert that ". . other animal studies in which TNF was used in conjunction with interferons showed a similar beneficial effect for melanomas and for carcinomas." I question the phrase "similar beneficial effect", when the results of those studies are compared with what can be achieved in the treatment of a truly sensitive tumour growing in a normal, immunocompetent syngeneic recipient. A properly timed, single-shot systemic treatment is generally sufficient to give longterm cures in $70-100 \%$ of the animals treated. Among the essential prerequisites for this phenomenon is the fact that the tumour must be immunogenic as classically defined ${ }^{3,4}$. As I implied ${ }^{2}$, the embryonic origin of the tumour may have no more importance than simply to help identify those tumours which have the highest probability of being immunogenic.

The clinical problem, of course, is that we have no comparable method of establishing the degree of immunogenicity of human tumours a priori, as we do with inbred mice. With humans, we must rather deal with a retrospective assessment, the essence of which has been provided for us in the medical records of Coley and his contemporaries. I believe that these records have already given us a perspective on which human tumours are likely to be immunogenic and which are not. Unfortunately, patient selection based on this perspective is decidedly imperfect; most inoperable mesodermally derived tumours were not known to have been cured by Coley's treatment (by the most optimistic estimate, only one in five). Matters are further complicated by the fact that even incurable, non-immunogenic murine tumours can be made to undergo some degree of necrosis in association with a therapeutic response that is only temporary ${ }^{3.4}$; Coley had made similar observations in the treatment of some human carcinomas. In contrast, because of the admirable follow-up efforts made by Coley's daughter Helen Coley Nauts, we know that a substantial number of inoperable soft-tissue sarcoma and lymphoma patients treated by her father and his contemporaries survived with no evidence of disease for more than 20 years (in my estimation, more analagous to the Meth A model). These records have given us a unique perspective that is essential in guiding us towards proper patient selection.

Finally, I specifically stated that "... serious consideration should be given to a return to an aggressive use of the vaccine ..."; not TNF-related or toxin-related therapy, but specifically the Coley vaccine itself. This is a point made in deference to the fact that the clinical accomplishments of Coley and his contemporaries were much beyond what we would be able to offer these same patients today. If we are going to make changes or improvisations in treatment, this should not be done until after we have at least managed to reproduce the original, basic observations.

\section{Charlie 0. Starnes}

Amgen, Inc.,

1840 Dehavilland Drive,

Thousand Oaks,

California 91320-1789, USA

\footnotetext{
Brouckaert, P, G. G., Fiers, W \& Lejeune, F. J Nature 358,630 (1992)

2. Starnes, C. O. Nature 357, 11-12 (1992).

3. Berendt, M. J. et al. J. exp. Med. 148, 1550-1559 (1978).

4. Berendt, M. J. et al. J. exp. Med. 148, 1560-1569 1978).
} 